\title{
SUBGROUPS OF MAPPING CLASS GROUPS RELATED TO HEEGAARD SPLITTINGS AND BRIDGE DECOMPOSITIONS
}

\author{
KEN'ICHI OHSHIKA AND MAKOTO SAKUMA
}

\begin{abstract}
Let $M=H_{1} \cup_{S} H_{2}$ be a Heegaard splitting of a closed orientable 3-manifold $M$ (or a bridge decomposition of a link exterior). Consider the subgroup $\mathrm{MCG}^{0}\left(H_{j}\right)$ of the mapping class group of $H_{j}$ consisting of mapping classes represented by auto-homeomorphisms of $H_{j}$ homotopic to the identity, and let $G_{j}$ be the subgroup of the automorphism group of the curve complex $\mathcal{C C}(S)$ obtained as the image of $\operatorname{MCG}^{0}\left(H_{j}\right)$. Then the group $G=\left\langle G_{1}, G_{2}\right\rangle$ generated by $G_{1}$ and $G_{2}$ preserve the homotopy class in $M$ of simple loops on $S$. In this paper, we study the structure of the group $G$ and the problem to what extent the converse to this observation holds.
\end{abstract}

Let $M$ be a closed orientable 3-manifold and $S$ a Heegaard surface of $M$. Then $M$ is decomposed into two handlebodies $H_{1}$ and $H_{2}$ such that $S=\partial H_{1}=\partial H_{2}$. We consider the (extended) mapping class group of $S$, i.e., the group of isotopy classes of (possibly orientation-reversing) autohomeomorphisms of $S$, and denote it by $\operatorname{MCG}(S)$. Let $\operatorname{MCG}\left(H_{j}\right)$ denote the mapping class group of $H_{j}(j=1,2)$. Then $\mathrm{MCG}\left(H_{j}\right)$ can be identified with a subgroup of $\mathrm{MCG}(S)$, by restricting an auto-homeomorphism of $H_{j}$ to $S$. We consider the subgroup of $\mathrm{MCG}\left(H_{j}\right)$ consisting of mapping classes represented by auto-homeomorphisms of $H_{j}$ homotopic to the identity, and denote it by $\mathrm{MCG}^{0}\left(H_{j}\right)$.

Now, let $\mathcal{C C}(S)$ be the curve complex of $S$, namely the simplicial complex each of whose vertex represents an isotopy class of essential simple closed curves in $S$ and each of whose simplex represents a set of isotopy classes with pairwise disjoint representatives. Then it is known that the natural action of $\operatorname{MCG}(S)$ on $\mathcal{C C}(S)$ induces a surjection from $\operatorname{MCG}(S)$ onto the simplicial automorphism group $\operatorname{Aut}(\mathcal{C C}(S))$ whose kernel is trivial or the cyclic group of order 2 generated by hyper-elliptic involution depending on whether the genus of $S$ is greater than or equal to 2 (see [9, Section 8], [13] and [20]). In this way, $\operatorname{MCG}(S)$ (or its quotient by the order 2 cyclic group) is canonically identified with $\operatorname{Aut}(\mathcal{C C}(S))$.

2010 Mathematics Subject Classification. Primary 57M50, 57M07, 30F40, 20F34

The first author was supported by JSPS Grants-in-Aid 22654008. The second author was supported by JSPS Grants-in-Aid 21654011. 
Let $G_{j}$ be the image of the subgroup $\operatorname{MCG}^{0}\left(H_{j}\right)$ of $\operatorname{MCG}(S)$ in $\operatorname{Aut}(\mathcal{C C}(S))$, and let $G=\left\langle G_{1}, G_{2}\right\rangle$ be the subgroup of $\operatorname{Aut}(\mathcal{C C}(S))$ generated by $G_{1}$ and $G_{2}$. Then $G$ preserves the homotopy classes in $M$ of the simple closed curves on $S$, namely, for any $g \in G$ and for any vertex $\alpha$ of $\mathcal{C C}(S), g \alpha$ is homotopic to $\alpha$ in $M$. (We ignore the distinction between a vertex of $\mathcal{C C}(S)$ and a simple closed curve in $S$ representing the vertex.)

Let $\Delta_{j}$ be the subset of the vertex set of $\mathcal{C C}(S)$ consisting of the meridians of $H_{j}$, namely the set of vertices in $\mathcal{C C}(S)$ represented by simple closed curves which bound discs in $H_{j}$. Let $Z$ be the set of vertices in $\mathcal{C C}(S)$ represented by simple closed curves which are null-homotopic in $M$. Then, by the above observation, the orbit $G\left(\Delta_{1} \cup \Delta_{2}\right)$ is contained in $Z$. The following natural question was posed by Minsky in [7, Question 5.4].

Question 0.1. When is $Z$ equal to the orbit $G\left(\Delta_{1} \cup \Delta_{2}\right)$ ?

The same question makes sense not only for Heegaard surfaces but also for bridge spheres as follows. Let $K$ be a knot or a link in $S^{3}$, and let $S$ be a bridge sphere of $K$. Then $\left(S^{3}, K\right)$ is a union of two trivial tangles $\left(B_{1}^{3}, t_{1}\right)$ and $\left(B_{2}^{3}, t_{2}\right)$ such that $(S, S \cap K)=\partial\left(B_{1}^{3}, t_{1}\right)=\partial\left(B_{2}^{3}, t_{2}\right)$. Here a trivial tangle means a pair of a 3 -ball $B^{3}$ and mutually disjoint arcs properly embedded in $B^{3}$ which are simultaneously parallel to mutually disjoint arcs in $\partial B^{3}$. We denote the punctured sphere $S-K$ by the same symbol $S$, and consider the (extended) mapping class group $\operatorname{MCG}(S)$ of the punctured sphere $S$. Then the mapping class group $\operatorname{MCG}\left(B_{j}^{3}, t_{j}\right)$ of the pair $\left(B_{j}^{3}, t_{j}\right)$ can be identified with a subgroup of $\mathrm{MCG}(S)$, by restricting an auto-homeomorphism of $\left(B_{j}^{3}, t_{j}\right)$ to $S$. Consider the subgroup of $\operatorname{MCG}\left(B_{j}^{3}, t_{j}\right)$ consisting of mapping classes represented by homeomorphisms pairwise-homotopic to the identity, and denote it by $\operatorname{MCG}^{0}\left(B_{j}^{3}, t_{j}\right)$. Let $G_{j}$ be the image of the subgroup $\operatorname{MCG}^{0}\left(B_{j}^{3}, t_{j}\right)$. of $\operatorname{MCG}(S)$ in $\operatorname{Aut}(\mathcal{C C}(S))$, and let $G=\left\langle G_{1}, G_{2}\right\rangle$ be the subgroup of Aut $(\mathcal{C C}(S))$ generated by $G_{1}$ and $G_{2}$. Let $\Delta_{j}$ be the set of vertices in $\mathcal{C C}(S)$ represented by simple closed curves which bound discs in $B_{j}^{3}-t_{j}$, and let $Z$ be the set of vertices in $\mathcal{C C}(S)$ represented by simple closed curves which are null-homotopic in the link complement $M:=S^{3}-K$. Then we can see that the orbit $G\left(\Delta_{1} \cup \Delta_{2}\right)$ is contained in $Z$. This observation was a starting point of [33], which gave rise to a systematic construction of epimorphisms between 2-bridge link groups. Again, it is natural to ask when $Z$ is equal to $G\left(\Delta_{1} \cup \Delta_{2}\right)$ (cf. [33, Question 9.1]).

In the second author's joint work with Donghi Lee [17, a complete answer to the above question for 2-bridge links was given. Moreover, the following results were obtained in a series of joint work [16, 17, 18, and they were applied in [19] to give a variation of McShane's identity for 2-bridge links.

Theorem 1. Let $K$ be a 2-bridge link in $S^{3}$ which is neither the trivial knot nor the 2-component trivial link, and let $S$ be a 2-bridge sphere of $K$. Let $G=\left\langle G_{1}, G_{2}\right\rangle, \Delta_{j}$, and $Z$ be as explained above, and let $\Lambda(G)$ and 
$\Omega(G)=\mathcal{P} \mathcal{M L}(S)-\Lambda(G)$, respectively, be the limit set and the domain of discontinuity of the action $G$ on $\mathcal{P} \mathcal{M L}(S)$. Then the following hold.

(1) The set $Z$ is equal to the orbit $G\left(\Delta_{1} \cup \Delta_{2}\right)$.

(2) The closure of $Z=G\left(\Delta_{1} \cup \Delta_{2}\right)$ in $\mathcal{P} \mathcal{M L}(S)$ is equal to the limit set $\Lambda(G)$. Moreover $\bar{Z}=\Lambda(G)$ has measure 0 in $\mathcal{P} \mathcal{M L}(S)$.

(3) The domain of discontinuity $\Omega(G)$ has full measure in $\mathcal{P} \mathcal{M L}(S)$, and no essential simple closed curve in $S$ representing a point in $\Omega(G)$ is null-homotopic in $M=S^{3}-K$.

(4) Suppose that $K$ is neither a torus link nor a twist knot. Then no essential simple closed curve in $S$ representing a point in $\Omega(G)$ is peripheral in $M$, i.e., no such simple closed curve is homotopic to a closed curve in a peripheral torus $\partial N(K)$ in $M$.

(5) Suppose that $K$ is neither a torus link nor the Whitehead link. Then, for any two essential simple closed curves in $S$ representing distinct points in $\Omega(G)$, they are homotopic in $M$ if and only if they lie in the same $G$-orbit.

(6) The group $G$ is isomorphic to the free product $G_{1} * G_{2}$.

It is natural to ask if the above theorem holds in a more general setting (see [35]). The purpose of this paper is to give the following partial answers to this natural question.

(1) If $S$ is a Heegaard surface or a bridge sphere with sufficiently high Hempel distance, then the subgroup $G=\left\langle G_{1}, G_{2}\right\rangle$ of $\operatorname{Aut}(\mathcal{C C}(S))$ is isomorphic to the free product $G_{1} * G_{2}$ (Theorem 2).

(2) If $S$ is a Heegaard surface, with $R$-bounded combinatorics for some $R>0$, of a closed orientable hyperbolic 3-manifold $M$ and if the Hempel distance of $S$ is larger than a constant $K_{0}$, depending only on the topological type of $S$ and the constant $R$, then there is a non-empty open set $O$ in the projective measured lamination space $\mathcal{P} \mathcal{M L}(S)$, such that

(a) no simple closed curve in $S$ representing a point in $O$ is nullhomotopic in $M$,

(b) two simple closed curves in $S$ representing distinct points in $O$ cannot be homotopic in $M$.

In particular, the action of $G$ on $\mathcal{P} \mathcal{M L}(S)$ has a non-empty domain of discontinuity (Theorems 3 and 4 ).

(3) Suppose that $M_{n}$ is obtained from two handlebodies by an $n$-time iteration of a generic pseudo-Anosov map $\phi$ and consider the Heegaard splitting of $M_{n}$ consisting of the two handlebodies. Then the subset $O$ in Theorem 3 for the Heegaard surface of $M_{n}$ can be made almost cover the entire projective lamination space so that the almost every point in the projective lamination space is contained in the open subset $O$ for $M_{n}$ with sufficiently large $n$ (Theorem 5 ).

We note that the result of Namazi [26] implies that if the Hempel distance of a Heegaard splitting $M=H_{1} \cup H_{2}$ is sufficiently large, then $G_{1} \cap G_{2}$ is 
finite. More generally, the result of Johnson [10] implies that $G_{1} \cap G_{2}$ is finite if the Hempel distance is greater than 3. Thus Theorem 2 may be regarded as a partial refinement of these consequences of the results of 26 ] and [10. We also note that Theorems 3 and 5 may be regarded as a variant of the asymptotical faithfulness of the homomorphism $\pi_{1}\left(H_{i}\right) \rightarrow \pi_{1}(M)$ established by Namazi [25, Theorem 1.6] and Namazi and Souto [28, Lemma 6.1 and Theorem 6.1].

The authors would like to thank Brian Bowditch for his essential contribution to the proof of Theorem 2, without which they should not have been able to complete the work. They would also like to thank Jeff Brock and Yair Minsky for stimulating conversation, valuable comments on the first version of this paper, and allowing them to read a draft of their joint work [3] with Hossein Namazi and Juan Souto on model manifolds, on which Theorems 3 and 4 depend.

\section{Structure of the group $\left\langle G_{1}, G_{2}\right\rangle$}

In this section, we shall prove the following theorem.

Theorem 2 (Bowditch-Ohshika-Sakuma). There is a constant $K_{0}$ depending only on the topological type of $S$ with the following property. For a Heegaard splitting or a bridge decomposition $M=H_{1} \cup_{S} H_{2}$ with its Hempel distance greater than $K_{0}$, the group $\left\langle G_{1}, G_{2}\right\rangle$ is decomposed into a free product $G_{1} * G_{2}$, where $G_{1}$ and $G_{2}$ are subgroups of $\operatorname{Aut}(\mathcal{C C}(S))$ defined in the introduction.

We recall the terminology in Gromov's theory of hyperbolic metric spaces. Let $X$ be a geodesic space, i.e. a metric space in which every pair of points can be connected by a geodesic segment. Let $\triangle=\overline{P Q} \cup \overline{Q R} \cup \overline{R P}$ be a geodesic triangle with its vertices $P, Q$ and $R$ in $X$. We consider a map, $\tau_{\triangle}$, from $\triangle$ to a "tripod" $T_{\triangle}$ which is an edge-wise isometry. We call this map the comparison map to $T_{\triangle}$. The map $\tau_{\triangle}$ has a property that two points $a \in \overline{P Q}$ and $b \in \overline{Q R}$ are identified under $\tau_{\triangle}$ if and only if $d(Q, a)=d(Q, b) \leq(P \mid R)_{Q}$, where the last term is the Gromov product defined by $(P \mid R)_{Q}=\frac{1}{2}(d(P, Q)+d(R, Q)-d(P, R))$. The same holds even if we permute $P, Q$ and $R$. Now, the triangle $\triangle$ is said to be $\delta$-thin when for each pair of points $a, b \in \triangle$ with $\tau_{\triangle}(a)=\tau_{\triangle}(b)$, we have $d_{X}(a, b) \leq \delta$. A geodesic space $X$ is said to be $\delta$-hyperbolic if every triangle in $X$ is $\delta$-thin.

In the following argument, we shall use the Gromov hyperbolicity of the curve complex $\mathcal{C C}(S)$ and the quasi-convexity of $\Delta_{1}$ and $\Delta_{2}$, where $\Delta_{1}$ and $\Delta_{2}$ are the subcomplexes of $\mathcal{C C}(S)$ spanned by the simple closed curves bounding disks in $H_{1}$ and $H_{2}$, respectively. (See Masur-Minsky [21] and 23.) Let $\delta$ be a positive constant such that $\mathcal{C C}(S)$ is $\delta$-hyperbolic. Let $L$ be a constant depending only on the topological type of $S$ such that both $\Delta_{1}$ and $\Delta_{2}$ are $L$-quasi-convex: any geodesic segment connecting two points in $\Delta_{1}\left(\operatorname{resp} . \Delta_{2}\right)$ lies in the $L$-neighbourhood of $\Delta_{1}\left(\right.$ resp. $\left.\Delta_{2}\right)$. 
Now, we start to prove that $\left\langle G_{1}, G_{2}\right\rangle$ is decomposed as $G_{1} * G_{2}$. Take a shortest geodesic segment $\gamma$ connecting $\Delta_{1}$ and $\Delta_{2}$ in $\mathcal{C C}(S)$, and denotes its endpoint in $\Delta_{1}$ by $x_{\gamma}$ and the one in $\Delta_{2}$ by $z_{\gamma}$. Consider a word $g_{1} h_{1} \ldots g_{p} h_{p}$, where $g_{j} \in G_{1}, h_{j} \in G_{2}$ and suppose that none of them is the identity. We show that the element of $\operatorname{Aut}(\mathcal{C C}(S))$ determined by the word $g_{1} h_{1} \ldots g_{p} h_{p}$ is nontrivial. In general, we need to consider the case where $g_{1}=1$ or $h_{p}=1$, but the argument needs no modification even in these cases.

Consider the translates $g_{1} \gamma$ of $\gamma$. Then the endpoint $x_{\gamma}$ of $\gamma$ and the endpoint $g_{1} x_{\gamma}$ of $g_{1} \gamma$ are both contained in $\Delta_{1}=g_{1} \Delta_{1}$. We connect $x_{\gamma}$ and $g_{1} x_{\gamma}$ by a geodesic segment $\delta_{1}$, which lies in the $L$-neighbourhood of $\Delta_{1}$. Next we consider the translate $g_{1} h_{1} \gamma$, and connect the endpoint $g_{1} z_{\gamma}$ of $g_{1} \gamma$ with the endpoint $g_{1} h_{1} z_{\gamma}$ of $g_{1} h_{1} \gamma$ by a geodesic segment $\delta_{1}^{\prime}$, which lies in the $L$-neighbourhood of $g_{1} \Delta_{2}=g_{1} h_{1} \Delta_{2}$. Repeating this process, we construct a piecewise geodesic arc $\alpha=\gamma \cup \delta_{1} \cup g_{1} \gamma \cup \delta_{1}^{\prime} \cup g_{1} h_{1} \gamma \cup \delta_{2} \cup g_{1} h_{1} g_{2} \gamma \cup \delta_{2}^{\prime} \cup$ $\cdots \cup \delta_{p}^{\prime} \cup g_{1} h_{1} \ldots g_{p} h_{p} \gamma$.

Let $d$ be either $\delta_{j}$ or $\delta_{j}^{\prime}$ in $\alpha$, and let $c$ be its preceding geodesic segment, that is, $g_{1} h_{1} \ldots h_{j-1} \gamma$ for $\delta_{j}$ or $g_{1} h_{1} \ldots g_{j} h_{j} \gamma$ for $\delta_{j}^{\prime}$. We connect the endpoints of $c \cup d$ by a geodesic segment, and denote it by $e$. Let $\triangle$ be the geodesic triangle $c \cup d \cup e$, and $\tau_{\triangle}: \triangle \rightarrow T_{\triangle}$ the comparison map to a tripod as explained above. Then the $L$-quasi-convexity of $\Delta_{1}, \Delta_{2}$ implies the following lemma.

Lemma 1.1. There is a constant $L^{\prime}$ depending only on $\delta$ and $L$ (hence only on the topological type of $S$ ) such that the longest subsegment of $d$ that is identified with a subsegment of $c$ under the map $\tau_{\triangle}$ has length at most $L^{\prime}$.

Proof. Set $x=c \cap d, y=d \cap e$ and $z=c \cap e$. By translating the entire picture so that $c$ becomes $\gamma$, we have only to consider the case where either $x, y$ lie in $\Delta_{1}$ and $z$ lies in $\Delta_{2}$ or $x, y$ lie in $\Delta_{2}$ and $z$ lies in $\Delta_{1}$. We may assume that $x$ and $y$ lie in $\Delta_{1}$, because we can argue in the same way also in the case where they lie in $\Delta_{2}$. Let $\ell$ be the length of the longest subsegment of $d$ which is identified with a subsegment of $c$ under $\tau_{\triangle}$, and let $p$ be its endpoints other than $x$. Then $d(x, p)=\ell$ and there is a point $q$ on $c$ such that $\tau_{\triangle}(p)=\tau_{\triangle}(q)$ and hence $d(p, q) \leq \delta$ by the $\delta$-thinness of the triangle $\triangle=c \cup d \cup e$. Since $q$ lies in the shortest geodesic segment $\gamma$ connecting $\Delta_{1}$ and $\Delta_{2}$, we have $d(q, x)=d\left(q, \Delta_{1}\right)$. Hence $\ell=d(q, x)=d\left(q, \Delta_{1}\right) \leq d(q, p)+d\left(p, \Delta_{1}\right) \leq \delta+L$. Thus, by setting $L^{\prime}$ to be $L+\delta$, we are done.

Next, let $f$ be the geodesic segment in $\alpha$ following $d$, and $h$ a geodesic segment connecting $e \cap c$ and the endpoint of $f$ other than $d \cap f$. We consider the geodesic triangle $e \cup f \cup h$, which we denote by $\triangle^{\prime}$, and the comparison map to its corresponding tripod $\tau_{\triangle^{\prime}}: \triangle^{\prime} \rightarrow T_{\triangle^{\prime}}$.

Lemma 1.2. There is a constant $L^{\prime \prime}$ depending only on the topological type of $S$ such that the longest subsegment of e identified with a subsegment of $f$ under the comparison map $\tau_{\triangle^{\prime}}$ has length at most $L^{\prime \prime}$. 
To prove this lemma, we shall use the following consequence of Bowditch's theorem established in [2] on acylindricity of the mapping class group action on the curve complex. The proof is deferred to the following section.

Proposition 1.3. For any $E>0$, there exists $L_{0}>0$ depending only on $E$ and the topological type of $S$ for which the following holds. Suppose that $\gamma$ contains a subsegment $\gamma^{\prime}$ with length at least $L_{0}$ and $g \in G_{i}$ such that $d(z, g z) \leq E$ for every $z \in \gamma^{\prime}$. Then $g$ is the identity.

Proof of Lemma 1.2 assuming Proposition 1.3. As was done in the proof of Lemma 1.1, we can assume that $f$ is $g_{1} \gamma$ and that $h$ connects the endpoint of $\gamma$ in $\Delta_{2}$ and that of $f$ in $g_{1} \Delta_{2}$. Let $f^{\prime}$ denote the longest subsegment of $f$ starting from $y=d \cap f$ that is identified with a subsegment of $e$ under the comparison map $\tau_{\triangle^{\prime}}$. We may assume length $\left(f^{\prime}\right)=L+2 \delta+K$ for some $K>0$, for otherwise the assertion of the lemma obviously holds.

First suppose that length $(d)>L+L^{\prime}+2 \delta$ for $L^{\prime}$ in Lemma 1.1. Let $w$ be a point on $f^{\prime}$ such that $d(y, w)$ is slightly bigger than $L+2 \delta$, which is guaranteed to exist since length $\left(f^{\prime}\right)=L+2 \delta+K>L+2 \delta$. Then there is a point $w^{\prime}$ lying on $e$ with $\tau_{\triangle^{\prime}}(w)=\tau_{\triangle^{\prime}}\left(w^{\prime}\right)$ and hence $d\left(w, w^{\prime}\right) \leq \delta$. By Lemma 1.1, the longest subsegment of $d$ starting from $y$ that is identified with a subsegment of $e$ under $\tau_{\triangle}$ has length greater than length $(d)-L^{\prime}$, which in turn is greater than $\left(L+L^{\prime}+2 \delta\right)-L^{\prime}=L+2 \delta$ by assumption. Thus we may assume, by choosing $w$ so that $L+2 \delta<d(y, w)<\operatorname{length}(d)-L^{\prime}$, that there is a point $w^{\prime \prime}$ on $d$ with $\tau_{\triangle}\left(w^{\prime}\right)=\tau_{\triangle}\left(w^{\prime \prime}\right), d\left(w^{\prime}, w^{\prime \prime}\right) \leq \delta$, and hence $d\left(w, w^{\prime \prime}\right) \leq 2 \delta$. By the $L$-quasi-convexity of $\Delta_{i}$, the distance from $w^{\prime \prime}$ to $\Delta_{i}$ is at most $L$. Therefore, we can connect $w$ by an arc of length at most $L+2 \delta$ to $\Delta_{1}$, which we denote by $\zeta$. The length of $\left.f \backslash f^{\prime}\right|_{[y, w]} \cup \zeta$ is less than length $(f)$, where $\left.f^{\prime}\right|_{[y, w]}$ denotes the subsegment of $f^{\prime}$ between $y$ and $w$. This contradicts the fact that $f$ is the shortest geodesic segment connecting $g_{1} \Delta_{2}$ to $\Delta_{1}$.

Next suppose that length $(d) \leq L+L^{\prime}+2 \delta$. Let $f^{\prime \prime}$ be the longest subsegment of $f$ identified with a subsegment of $e$ under $\tau_{\triangle^{\prime}}$ and then with that of $c$ under $\tau_{\triangle}$. Then length $\left(f^{\prime \prime}\right)>\operatorname{length}\left(f^{\prime}\right)-\operatorname{length}(d) \geq$ $(L+2 \delta+K)-\left(L+L^{\prime}+2 \delta\right) \geq K-L^{\prime}$. Let $c^{\prime \prime}$ be the subsegment of $c$ identified with $f^{\prime \prime}$ as in the above, and let $\varphi: c^{\prime \prime} \rightarrow f^{\prime \prime}$ be the isometry arising from the identification. Let $x^{\prime \prime}$ be the endpoint of $c^{\prime \prime}$ nearer to $x$. Then $d\left(x, x^{\prime \prime}\right) \leq L^{\prime}$ by Lemma 1.1, and hence $d\left(g_{1}(x), g_{1}\left(x^{\prime \prime}\right)\right) \leq L^{\prime}$. Thus $g_{1}\left(x^{\prime \prime}\right)$ is contained in the component of $f \backslash f^{\prime \prime}$ containing $y=g_{1}(x)$. Since the length of the component of $f \backslash f^{\prime \prime}$ is less than that of $d$, we have $d\left(g_{1}\left(x^{\prime \prime}\right), \varphi\left(x^{\prime \prime}\right)\right)<\operatorname{length}(d) \leq L+L^{\prime}+2 \delta$. Since both $g_{1}$ and $\varphi$ are isometries into $f$, this implies that $d\left(g_{1}(\xi), \varphi(\xi)\right)<L+L^{\prime}+2 \delta$ for every $\xi \in c^{\prime \prime}$. Hence we have $d\left(\xi, g_{1}(\xi)\right) \leq d(\xi, \varphi(\xi))+d\left(\varphi(\xi), g_{1}(\xi)\right)<2 \delta+L+L^{\prime}+2 \delta$ for every $\xi \in c^{\prime \prime}$. Therefore, we see by Proposition 1.3 that there is a constant $L_{0}$ depending only on $S$ which bounds length $\left(c^{\prime \prime}\right)=\operatorname{length}\left(f^{\prime \prime}\right)>K-L^{\prime}$ from above. Thus, $K$ is bounded by a constant $K^{\prime}$ depending only on $S$. By setting $L^{\prime \prime}$ to be $L+2 \delta+K^{\prime}$, we are done. 
Proposition 1.4. There are constants $A, B$ depending only on $L$ and $\delta$ such that $\alpha$ is an $(A, B)$-quasi-geodesic if $K_{0}$ is large enough.

Proof of Proposition 1.4. We shall first show that there are $A, B$ as above such that $c \cup d \cup f$ is an $(A, B)$-quasi-geodesic. By Lemma 1.1, except for geodesic segments starting from $x$ of lengths at most $L^{\prime}$, one on $c$ and the other on $d$, each point of $c \cup d$ is within the distance $\delta$ from $e$. In the same way, we see that by Lemma 1.2, except for geodesic segments starting from $y$ of length $L^{\prime \prime}$, one on $e$ and the other on $f$, every point on $e \cup f$ is within the distance $\delta$ from $h$. These imply that $c \cup d \cup f$ is a $\left(1,2 L^{\prime}+2 L^{\prime \prime}+4 \delta\right)$ quasi-geodesic.

This holds for every three consecutive arcs constituting $\alpha$ that has a translate of $\delta$ or $\delta^{\prime}$ in the middle. Now, in general in a $\delta$-hyperbolic geodesic space, for any $(C, D)$ there are $(A, B)$ and $l$ such that an arc each of whose subarc of length less than $l$ is $(C, D)$-quasi-geodesic is always $(A, B)$-quasigeodesic itself, where $A, B$ and $l$ depend only on $C, D$ and $\delta$. (See [6, Chap. 3, Théorème 1.4] or [4, Chap. III, Theorem 1.13].) Therefore, by taking $K_{0}$, which bounds the length of $\gamma$ from below, to be large enough, we see that there are $A, B$ depending only on $\delta$ and $L$ such that $\alpha$ is an $(A, B)$-quasigeodesic.

Let $\alpha^{\prime}$ be the subarc of $\alpha$ obtained by deleting the last geodesic segment $g_{1} h_{1} \ldots g_{p} h_{p} \gamma$ from $\alpha$. Proposition 1.4 implies that there is a constant $C$ depending only on $A$ and $B$ such that the endpoints of $\alpha^{\prime}$, which are $x_{\gamma}$ and $g_{1} h_{1} \ldots g_{p} h_{p} x_{\gamma}$, cannot be the same if length $(\gamma) \geq C$. Therefore, assuming $K_{0}$ to be greater than $C$, we see that the word $g_{1} h_{1} \ldots g_{p} h_{p}$ represents a non-identity element. As is noticed at the beginning of the proof, the same conclusion holds when $g_{1}=1$ or $h_{p}=1$. Hence $G=G_{1} * G_{2}$. This completes the proof of Theorem 2 .

\section{Bowditch'S THEOREM AND ITS CONSEQUENCE}

In this section, we shall prove Proposition 1.3 by using the following acylindricity of the mapping class group action on the curve complex proved by Bowditch [2].

Theorem 2.1 (Bowditch [2]). For any given $D>0$, there are $R>0$ and $a$ positive integer $N$ depending only on $D$ and the topological type of $S$ with the following property. Let $x, y$ be two points in $\mathcal{C C}(S)$ with $d(x, y) \geq R$. Then there are at most $N$ elements $g$ of $\operatorname{MCG}(S)$ such that both $d(x, g x) \leq D$ and $d(y, g y) \leq D$.

Before starting the proof of Corollary 1.3, we prepare the following lemma.

Lemma 2.2. There exists a constant $A>0$ depending only on $Q$ and $\delta$ for which the following holds. Let $Y$ be a $Q$-quasi-convex set in a $\delta$-hyperbolic geodesic space $X$ and $\pi: X \rightarrow Y$ the nearest point retraction. Then for any $B>0$, there exists $C>0$, depending only on $Q, \delta$ and $B$, such that for any 
points $z, w \in X$ with $d(z, w) \leq B$ and $d(z, Y) \geq C, d(w, Y) \geq C$, we have $d(\pi(z), \pi(w)) \leq A$.

Proof. This is a consequence of the $\delta$-thinness of triangles in $X$. We let $A$ be $2 Q+3 \delta+1$, and for given $B$, we set $C=B+Q+2 \delta+1$. Let $z, w$ be points in $X$ with $d(z, w) \leq B$ and $d(z, Y) \geq C, d(w, Y) \geq C$. For two points in $X$, we shall denote a geodesic segment connecting them (which we choose) by putting bar over them. We have only to show that length $(\overline{\pi(z) \pi(w)}) \leq A$.

Suppose not. Then there is a point $p \in \overline{\pi(z) \pi(w)}$ with $d(p, \pi(z)) \geq$ $Q+\delta+1 / 2$ and $d(p, \pi(w)) \geq Q+2 \delta+1 / 2$. We consider two geodesic triangles $\triangle_{w}=\overline{z w} \cup \overline{w \pi(w)} \cup \overline{z \pi(w)}$ and $\triangle_{z}=\overline{z \pi(z)} \cup \overline{\pi(z) \pi(w)} \cup \overline{z \pi(w)}$. By the $\delta$-thinness of $\triangle_{z}$, there is a point $q$ on either $\overline{z \pi(z)}$ or $\overline{z \pi(w)}$ with $\tau_{\triangle_{z}}(q)=\tau_{\triangle_{z}}(p)$ and $d(p, q) \leq \delta$. Suppose that $q$ lies on $\overline{z \pi(z)}$. Then $d(q, \pi(z))=d(q, Y) \leq d(q, p)+d(p, Y) \leq \delta+Q$. On the other hand, since $\tau_{\triangle_{z}}(q)=\tau_{\triangle_{z}}(p)$, we have $d(q, \pi(z))=d(p, \pi(z)) \geq Q+\delta+1 / 2$. This is a contradiction. This contradicts the assumption Thus we see that $q$ lies on $\overline{z \pi(w)}$.

Now we turn to consider the triangle $\triangle_{w}$. There is a point $r$ on either $\overline{z w}$ or $\overline{w \pi(w)}$ such that $\tau_{\Delta_{w}}(q)=\tau_{\Delta_{w}}(r)$ and hence $d(q, r) \leq \delta$ by the $\delta$ thinness of $\triangle_{w}$. Suppose first that $r$ lies on $\overline{z w}$. Then $d(r, w) \leq d(z, w) \leq B$. Therefore, we have $d(w, Y) \leq d(w, r)+d(r, q)+d(q, p)+d(p, Y) \leq B+2 \delta+Q$. This contradicts the assumption that $d(w, Y) \geq C=B+Q+2 \delta+1$.

Suppose next that $r$ lies on $\overline{w \pi(w)}$. Then $d(r, \pi(w))=d(r, Y) \leq d(r, q)+$ $d(q, p)+d(p, Y) \leq 2 \delta+Q$. On the other hand, the equalities $\tau_{\triangle_{w}}(r)=$ $\tau_{\triangle_{w}}(q), \tau_{\triangle_{z}}(q)=\tau_{\triangle_{z}}(p)$ imply $d(r, \pi(w))=d(q, \pi(w))=d(p, \pi(w)) \geq Q+$ $2 \delta+1 / 2$. This is a contradiction. Thus we have proved that $d(\pi(z), \pi(w)) \leq$ A.

Proof of Proposition 1.3. Recall that $\Delta_{i}(i=1,2)$ is $L$-quasi-convex. This implies that for $r \geq L$, the $r$-neighbourhood, $N_{r}\left(\Delta_{i}\right)$, of $\Delta_{i}$ is $2 \delta$-quasiconvex. (See [6, Chap 10, Proposition 1.2].) Let $\pi_{r}: \mathcal{C C}(S) \rightarrow N_{r}\left(\Delta_{i}\right)$ be a nearest point projection. Letting $Q$ in Lemma 2.2 be $2 \delta$, we get a constant $A>0$, which satisfies the following condition: For any $B>0$, there exists $C>0$, such that for any points $z, w \in \mathcal{C C}(S)$ with $d(z, w) \leq B$ and $d\left(z, N_{r}\left(\Delta_{i}\right)\right) \geq C, d\left(w, N_{r}\left(\Delta_{i}\right)\right) \geq C$, we have $d\left(\pi_{r}(z), \pi_{r}(w)\right) \leq A$ for every $r \geq L$.

Regarding this $A$ as $D$ in Theorem 2.1, we get a constant $R$ and a positive integer $N$, which satisfy the following condition: For two points $x, y$ in $\mathcal{C C}(S)$ with $d(x, y) \geq R$, there are at most $N$ elements $g$ of $\operatorname{MCG}(S)$ such that both $d(x, g x) \leq A$ and $d(y, g y) \leq A$.

By setting $B=(N+1) E$ for a given $E$ in our statement in Proposition 1.3 and recalling the choice of the constant $A$ using Lemma 2.2, we obtain a constant $C$ which satisfies the following condition: For any points $z, w \in$ $\mathcal{C C}(S)$ with $d(z, w) \leq B$ and $d\left(z, N_{r}\left(\Delta_{i}\right)\right) \geq C, d\left(w, N_{r}\left(\Delta_{i}\right)\right) \geq C$, we have $d\left(\pi_{r}(z), \pi_{r}(w)\right) \leq A$. Here $r$ is any real number with $r \geq L$. 
After this preparation, we now prove the conclusion of our proposition holds if we let $L_{0}$ be $L+C+R$. Suppose on the contrary that there is a subsegment $\gamma^{\prime}$ of $\gamma$ of length $L_{0}$ on which a non-trivial element $g \in G_{i}$ translates points within the distance $E$. Let $\xi$ be the point on $\gamma^{\prime}$ farthest from $\Delta_{i}$. Then $d\left(\xi, \Delta_{i}\right) \geq L_{0}=L+C+R$. Thus, for each $r$ with $L \leq$ $r \leq L+R$, we have $d\left(\xi, N_{r}\left(\Delta_{i}\right)\right) \geq C$. On the other hand, for each $j$ with $1 \leq j \leq N+1$, we have $d\left(\xi, g^{j}(\xi)\right) \leq j E \leq(N+1) E=B$. Hence $d\left(\pi_{r}(\xi), \pi_{r}\left(g^{j}(\xi)\right)\right) \leq A$ for each $r$ with $L \leq r \leq L+R$. Note that we may choose $\pi_{r}$ so that $\pi_{r}\left(g^{j}(\xi)\right)=g^{j}\left(\pi_{r}(\xi)\right)$. Thus we have $d\left(\pi_{r}(\xi), g^{j}\left(\pi_{r}(\xi)\right)\right) \leq$ $A$ for each $r$ with $L \leq r \leq L+R$ and for each $j$ with $1 \leq j \leq N+1$. Since we can assume that $\pi_{r}(\bar{\xi})$ lies on $\gamma$, this shows that there is a subsegment $\gamma^{\prime \prime}$ of $\gamma$ with length $R$ on which all points are translated within the distance $A$ by any $g^{j}$ for $j=1, \ldots N+1$. Since any element in $G_{i}$ has infinite order as was shown in the proof of Proposition 1.7 in Otal [34], we see that $g, \ldots, g^{N+1}$ are all distinct. This contradicts Theorem 2.1 .

\section{Non-TRIVIAL CURVES}

In this section and the next, we only consider Heegaard splittings, for our argument relies on the construction of model manifolds for Heegaard splittings due to Namazi [25, 27] and its generalisation by Namazi-Souto [28] and Brock-Minsky-Namazi-Souto [3]. We believe that we can obtain the same result for bridge decompositions since their theory is valid in more general settings including the case where the hyperbolic manifolds have torus cusps as is suggested in [3].

Before stating the theorem, we shall review the definitions of subsurface projections due to Masur-Minsky [22] and of bounded combinatorics introduced by Namazi [25]. Let $S$ be a closed surface and $Y$ a connected open incompressible subsurface of $S$, which is either an annulus or has negative Euler characteristic. The curve complex $\mathcal{C C}(Y)$ is defined in the same way as $\mathcal{C C}(S)$ unless $Y$ is either a once-punctured sphere or four-times punctured sphere or an annulus. When $Y$ is either a once-punctured torus or a four-times punctured sphere, $\mathcal{C C}(Y)$ is a one-dimensional simplicial complex whose vertices are the isotopy classes of essential simple closed curves and where two vertices are connected if their geometric intersection number is the least possible: 1 when $Y$ is a once-punctured torus and 2 when $Y$ is a four-times punctured sphere. When $Y$ is an annulus, we consider its compactification $\bar{Y}$, and $\mathcal{C C}(Y)$ is defined to be a one-dimensional simplicial complex whose vertices are the isotopy classes relative to the endpoints of essential arcs and where two vertices are connected if they are realised to be disjoint.

Let $c$ be an essential simple closed curve on $S$, and isotope it so that Fr $Y \cap c$ is transverse and there are no inessential intersection in $Y \cap c$. If $Y$ has negative Euler characteristic, we consider, roughly speaking, all possible essential simple closed curves obtained by connecting endpoints of $F \cap c$ 
by $\operatorname{arcs}$ on $\operatorname{Fr} Y$, and denote the set consisting of such simple closed curves by $\pi_{Y}(c)$ regarding it as a subset of $\mathcal{C C}(Y)$. If there are no such simple closed curves, we define $\pi_{Y}(c)$ to be the empty set. When $Y$ is an annulus, $\pi_{Y}(c)$ is defined to be the set of points in $\mathcal{C C}(Y)$ determined by the lifts of $c$ to the covering of $S$ associated to $\pi_{1}(Y)$ and its natural compactification as a hyperbolic surface. For a subset $C$ of $\mathcal{C C}(S)$, we define $\pi_{Y}(C)$ to be $\cup_{c \in C} \pi_{Y}(c)$. Also, we can define the projections of multi-curves and clean markings in the same way. See [22, Section 2] for precise definition.

Definition 3.1. Let $M=H_{1} \cup H_{2}$ be a Heegaard splitting along $S$ and let $\Delta_{1}$ and $\Delta_{2}$ be the subsets of $\mathcal{C C}(S)$ consisting of the meridians of $H_{1}$ and $H_{2}$, respectively. For a positive real number $R$, we say that the decomposition has $R$-bounded combinatorics if there are handlebody pants decompositions $P_{1} \subset \Delta_{1}$ and $P_{2} \subset \Delta_{2}$ of $H_{1}$ and $H_{2}$, such that the distance between $P_{1}$ and $P_{2}$ in $\mathcal{C C}(S)$ is equal to the distance between $\Delta_{1}$ and $\Delta_{2}$, and that the distance in $\mathcal{C C}(Y)$ between $\pi_{Y}\left(P_{1}\right)$ and $\pi_{Y}\left(P_{2}\right)$ is bounded by $R$ for any proper incompressible subsurface $Y$ of $S$ which is either an annulus or has negative Euler characteristic.

Theorem 3. For any given positive constant $R$, there is a constant $K_{0}$ depending only on $R$ and the topological type of $S$ with the following property. If a closed hyperbolic 3-manifold $M$ has a Heegaard splitting $M=H_{1} \cup_{S}$ $\mathrm{H}_{2}$ with $\mathrm{R}$-bounded combinatorics whose Hempel distance is greater than or equal to $K_{0}$, then there is a non-empty open set $O$ in $\mathcal{P} \mathcal{M L}(S)$ such that no simple closed curves in $S$ representing a point in $O$ is null-homotopic in $M$. Furthermore, two simple closed curves in $S$ representing distinct points in $O$ cannot be homotopic in $M$.

In the following argument, we fix a homeomorphism type of $S$ once and for all.

As is noted in the above, our proof of this theorem relies on the work of Namazi [25, 27] and its generalisation by Brock-Minsky-Namazi-Souto 3] on model manifolds of Heegaard splittings and more complicated glueing. They showed that for a given positive constant $R$, if we take a sufficiently large $K_{0}$, then for any 3-manifold $M$ which admits a Heegaard splitting $M=H_{1} \cup_{S} H_{2}$ with $R$-bounded combinatorics and Hempel distance $\geq K_{0}$, there is a bi-Lipschitz model manifold which is a pinched negatively curved 3-manifold homeomorphic to $M$ with its pinching constants depending only on $K_{0}$ and the topological type of $S$, and is obtained by pasting hyperbolic handlebodies as will be explained below.

The model manifold has a negatively curved metric obtained by glueing hyperbolic metrics on $H_{1}$ and $H_{2}$ using a hyperbolic 3-manifold homeomorphic to $S \times \mathbb{R}$. We shall explain how to do this following the description by Namazi [25]. We first choose handlebody pants decompositions $P_{1} \subset \Delta_{1}$ and $P_{2} \subset \Delta_{2}$ of $H_{1}$ and $H_{2}$ as in Definition 3.1, which realises the distance between $\Delta_{1}$ and $\Delta_{2}$. By [25, Lemma 2.9], there are clean markings $\alpha_{1}, \alpha_{2}$ with base curves $P_{1}, P_{2}$ respectively such that $\pi_{Y}\left(\alpha_{1}\right)$ and $\pi_{Y}\left(\alpha_{2}\right)$ are within 
the distance $R$ in $\mathcal{C C}(Y)$ for any proper open incompressible subsurface $Y$ of $S$. We take points $m_{1}, m_{2}$ in the Teichmüller space of $S$ such that the total length of $\alpha_{j}$ with respect to the hyperbolic metric compatible with $m_{j}$ is shortest among all clean markings. Let $g_{1}$ and $g_{2}$ be convex cocompact hyperbolic metrics on Int $H_{1}$ and Int $H_{2}$ whose marked conformal structures at infinity are $m_{2}$ and $m_{1}$ respectively, where we define the markings of $H_{1}$ and $H_{2}$ by regarding them as being embedded in $M$ with the common boundary $S$. Then if we take $K_{0}$ to be sufficiently large, there are a doubly degenerate hyperbolic 3-manifold $M_{S}$ homeomorphic to $S \times \mathbb{R}$ and open sets $U_{1} \subset \operatorname{Int} H_{1}, U_{2} \subset$ Int $H_{2}, V_{1}, V_{2} \subset M_{S}$ with the following conditions.

(a) The hyperbolic 3 -manifold $M_{S}$ has $\epsilon_{0}$-bounded geometry with $\epsilon_{0}$ depending only on $R$; that is, every closed geodesic in $M_{S}$ has length greater than or equal to $\epsilon_{0}$.

(b) All of $U_{1}, U_{2}, V_{1}, V_{2}$ are homeomorphic to $S \times(0,1)$.

(c) There is a bi-Lipschitz diffeomorphism between the subspace $U_{j}$ of the hyperbolic manifold (Int $H_{j}, g_{j}$ ) and the subspace $V_{j}$ of the hyperbolic manifold $M_{S}$ for $j=1,2$ which tends to an isometry uniformly in the $C^{2}$-topology as $K_{0} \rightarrow \infty$.

(d) The intersection $V_{1} \cap V_{2}$ is also homeomorphic to $S \times(0,1)$.

(e) We define the width of $V_{1} \cap V_{2}$ to be the distance between its two frontier components with respect to the metric of $M_{S}$. Then, the width of $V_{1} \cap V_{2}$ goes to $\infty$ as $K_{0} \rightarrow \infty$.

These hold because of the following facts. Suppose that we are given a sequence of Heegaard splittings as above with Hempel distance going to $\infty$. We use the superscript $i$ to denote the $i$-th pants decompositions and metrics, as $P_{j}^{i}$ or $m_{j}^{i}$ or $g_{j}^{i}$ for $j=1,2$. If we fix a marking on $H_{1}$, then the convex cocompact hyperbolic structure (Int $H_{1}, g_{1}^{i}$ ) converges to a geometrically infinite hyperbolic structure $g_{1}^{\infty}$ in Int $H_{1}$ both algebraically and geometrically, after passing to a subsequence. The convergence is guaranteed by the facts that the total length of $P_{2}^{i}$, with respect to the hyperbolic metric $m_{2}^{i}$ on $S$ corresponding to the conformal structure at infinity associated with $g_{1}^{i}$, is uniformly bounded and that $\left\{P_{2}^{i}\right\}$ converges to a lamination in the Masur domain of the projective lamination space passing to a subsequence. The hyperbolic 3-manifold ( $\operatorname{Int} H_{1}, g_{1}^{\infty}$ ) is asymptotically isometric to a doubly degenerate hyperbolic 3 -manifold $M_{S}$ whose ending laminations are the limits of $\left\{P_{1}^{i}\right\}$ and $\left\{P_{2}^{i}\right\}$ respectively; that is, for any $\delta>0$, there exists a compact set $K$ such that Int $H_{1} \backslash K$ with the metric $g_{1}^{\infty}$ is embedded into a neighbourhood of the end of $M_{S}$, with ending lamination equal to the limit of $\left\{P_{2}^{i}\right\}$, by a diffeomorphism which is $\delta$-close to an isometry in the $C^{2}$-topology. Combined with the convergence which we have just explained, it follows that for any $\delta>0$, there exist a compact set $K$ and a sequence $\left\{R_{i}\right\}$ going to $\infty$ such that for the $R_{i}$-neighbourhood $N_{R_{i}}(K)$ of $K$ in Int $H_{1}$ with respect to the metric $g_{1}^{i}$, its subset $N_{R_{i}}(K) \backslash K$ is embedded into $M_{S}$ by a diffeomorphism $\delta$-close to an isometry. In the same 
way, (Int $H_{2}, g_{2}^{\infty}$ ) is asymptotically isometric to $M_{S}$, and the complement of a compact set in (Int $\mathrm{H}_{2}, g_{2}^{\infty}$ ) is embedded nearly isometrically to a neighbourhood of the other end of $M_{S}$. These facts guarantee the existence of the sets $U_{1} \subset \operatorname{Int} H_{1}, U_{2} \subset \operatorname{Int} H_{2}, V_{1}, V_{2} \subset M_{S}$ which appeared above. (As for more details on the proof of this convergence and the property of its limit, refer to 32 and [29].) We also note that the bi-Lipschitz diffeomorphisms in (c) are liftable as can be seen by checking the conditions in 24, Lemma 3.1].

Now the model manifold is constructed as follows. We paste (Int $H_{1}, g_{1}$ ) and (Int $H_{2}, g_{2}$ ) to get a manifold homeomorphic to $M$, identifying $U_{1}$ with $V_{1}$ and $U_{2}$ with $V_{2}$ by bi-Lipschitz diffeomorphisms close to isometries defined above, letting $V_{1} \cap V_{2}$ be the margin of pasting and throwing away neighbourhoods of ends outside $U_{1}$ and $U_{2}$. Construct a Riemannian metric on the resulting manifold by glueing the hyperbolic metrics $g_{1}$ and $g_{2}$ along the margin of glueing by using a bump function. Then, since both $g_{1}$ and $g_{2}$ get closer and closer to the hyperbolic metric of $M_{S}$ on a neighbourhood of the margin of glueing, the sectional curvature of the resulting metric lies between $(-1-\varepsilon,-1+\varepsilon)$, with $\varepsilon \rightarrow 0$ as $K_{0} \rightarrow \infty$. Tian's theorem [37] (cf. [25, Chapter 12]) implies that this metric is $\rho$-close (as Riemannian metric) to the original hyperbolic metric of $M$ in the $C^{2}$-topology, where the constant $\rho$ depends only on $K_{0}$ and goes to 0 as $K_{0} \rightarrow \infty$.

In the following, we show that if we take the lower bound $K_{0}$ of the Hempel distance to be large enough, then there is an open set $O$ in $\mathcal{P} \mathcal{M L}(S)$ satisfying the conditions in Theorem 3. As was explained above, the hyperbolic metric on $M$ is uniformly close to the constructed negatively curved model metric in the $C^{2}$-topology. On the other hand, as was shown in the construction, the negatively curved metric on $M$ is close to the hyperbolic metric on $M_{S}$ in the part corresponding to $V_{1} \cap V_{2}$ which appeared above. Recall that, for any positive constant $\epsilon$, there is an upper bound (depending only on $\epsilon$ and the topological type of $S$ ) for the diameters modulo their $\epsilon$-thin parts of the pleated surfaces in $M_{S}$ intersecting $V_{1} \cap V_{2}$ (see [36. Chapter 9] and [30. Lemma 1.2]). Since $M_{S}$ has $\epsilon_{0}$-bounded geometry with $\epsilon_{0}$ depending only on $R$ in our case, the injectivity radii on pleated surfaces are bounded from below by $\epsilon_{0} / 2$. Therefore, there is a constant $K$, depending only on $R$ and the topological type of $S$, such that a pleated surface in $M_{S}$ which has a point in $V_{1} \cap V_{2}$ at the distance at least $K$ from the frontier of $V_{1} \cap V_{2}$ must be entirely contained in $V_{1} \cap V_{2}$.

Now, recall that Thurston defined a notion of rational depth of measured laminations (see [36, Definition 9.5.10]). A measured lamination is said to have rational depth $k$ when it is carried by a train track with a weight system $w$ which has $k$ independent linear relations over $\mathbb{Q}$ in addition to those coming from the switch conditions. Thurston proved the following two facts: (1) The set of measured laminations of rational depth 0 has full measure in $\mathcal{M L}(S)$. (2) For any two measured laminations, we can find an embedded arc in $\mathcal{M L}(S)$ connecting them whose interior passes only 
measured laminations of rational depth less than 2, and only countably many measured laminations of rational depth 1 .

One more thing proved by Thurston which we need to use now is the existence of one-parameter family consisting of pleated surfaces and negatively curved interpolated surfaces between two pleated surfaces (see [36. Section 9.5] and [30, Section 4.E]). This shows that for any point in $M_{S}$, there is a negatively curved surface in the family passing through that point and that such a surface is contained in a uniformly bounded neighbourhood of a pleated surface realising a measured lamination of rational depth 0 . Therefore, any point in $M_{S}$ has a pleated surface homotopic to the inclusion of $S$, which realises a measured lamination of rational depth 0 , which contains a point within a uniformly bounded distance from the give point. In particular, if the width of $V_{1} \cap V_{2}$ is large enough, then there is a pleated surface $f$ realising a measured lamination $\lambda$ of rational depth- 0 whose image is contained in $V_{1} \cap V_{2}$ and is away from the frontier of $V_{1} \cap V_{2}$ by the distance greater than $L$ for any $L$ sufficiently smaller than the width of $V_{1} \cap V_{2}$. We shall specify this constant $L$ later. For the moment, we just note that we can take $L$ to be large if $K_{0}$ is large. Since we can take $V_{1}$ and $V_{2}$ so that the width of $V_{1} \cap V_{2}$ goes to $\infty$ as the lower bound of Hempel distance $K_{0} \rightarrow \infty$, we may assume, by taking $K_{0}$ large enough, that such a pleated surface $f$ realising a measured lamination $\lambda$ of rational depth- 0 actually exists.

We shall show by contradiction that there is an open neighbourhood $O$ of $[\lambda]$ in $\mathcal{P} \mathcal{M L}(S)$ such that every lamination contained in $O$ is realisable by a pleated surface in $V_{1} \cap V_{2}$ whose image is at the distance greater than $L$ from the frontier of $V_{1} \cap V_{2}$. Suppose that such an open set does not exist. Since the realisability of a measured lamination is invariant under scalar multiplications, there is a sequence of measured laminations $\left\{\lambda_{i}\right\}$ converging to $\lambda$, which cannot be realised by pleated surfaces contained in $V_{1} \cap V_{2}$ at the distance greater than $L$ from the frontier of $V_{1} \cap V_{2}$. Since there are only two unmeasured laminations which are unrealisable in $M_{S}$ and the measured laminations having these laminations as supports constitute closed subsets of $\mathcal{M L}(S)$ not containing $\lambda$, by taking a subsequence, we can assume that all the $\lambda_{i}$ can be realised by pleated surfaces in $M_{S}$. Let $f_{i}: S \rightarrow M_{S}$ be a pleated surface realising $\lambda_{i}$. Since $\left\{\lambda_{i}\right\}$ converges to $\lambda$, which does not represent an ending lamination, we see that there is a compact set which all the images of $f_{i}$ intersect. Therefore, by passing to a subsequence, $\left\{f_{i}\right\}$ converges uniformly to a pleated surface $f_{\infty}$ realising $\lambda$ (cf. [5, Theorem $5.2 .18])$.

Since $\lambda$ has rational depth 0 , its realisation is unique; hence $f_{\infty}$ coincides with the pleated surface $f$ which appeared above. It follows that the image of $f_{i}$ is also contained in $V_{1} \cap V_{2}$ and at the distance greater than $L$ from the frontier of $V_{1} \cap V_{2}$, for sufficiently large $i$. This is a contradiction. Thus we have shown that there is an open set $O$ containing $[\lambda]$ and consisting of measured laminations which can be realised by pleated surfaces in $V_{1} \cap V_{2}$ whose images are at the distance greater than $L$ from the frontier of $V_{1} \cap V_{2}$. 
Next we shall show that no curve contained in $O$ can be null-homotopic in $M$ if we take the constant $K_{0}$ (and hence also $L$ ) to be large enough and make $O$ smaller accordingly. Suppose that a simple closed curve $c$ on $S$ is contained in $O$. Since $c$ is realised by a pleated surface contained in $V_{1} \cap V_{2}$, there is a closed geodesic $c^{*}$ in $V_{1} \cap V_{2}$ homotopic to $c$. Recall that there is a $C$-bi-Lipschitz diffeomorphism from an open set $V^{\prime}$ in $M$ to $V_{1} \cap V_{2}$, which lifts to a $C$-bi-Lipschitz diffeomorphism from the universal cover $\tilde{V}^{\prime}$ of $V^{\prime}$ to $\mathbb{H}^{3}$ (with respect to the path metrics), with a constant $C$ depending only on $S$ and $K_{0}$ and going to 1 as $K_{0} \rightarrow \infty$. Regard $c^{*}$ as a closed geodesic arc, and let $\tilde{c}$ be a geodesic arc in the universal cover $\widetilde{V_{1} \cap V_{2}} \subset \tilde{M}_{S}=\mathbb{H}^{3}$ obtained as a lift of the geodesic arc $c^{*}$. Then $\tilde{c}$ is pulled back to a $\left(C^{2}, 0\right)$-quasi-geodesic $\tilde{c}^{\prime}$ in the universal cover $\tilde{V}^{\prime}$ of $V^{\prime}$ (see [31, Lemma 2.36]). By the stability of quasi-geodesics (see [6, Chaptire 3 , Théorème 1.2] or [31, Theorem 2.31]), there is a constant $N$ depending only on $C$, which goes to 0 as $C \rightarrow 1$ such that $\tilde{c}^{\prime}$ can be homotoped to a geodesic arc fixing the endpoints within the $N$-neighbourhood of $\tilde{c}^{\prime}$ if $\tilde{c}^{\prime}$ is at the distance greater than $N$ from the ends of $\tilde{V}^{\prime}$.

To be more precise, we cannot directly use the stability which is valid only in a Gromov hyperbolic space, whereas our space $\tilde{V}^{\prime}$ is only a locally Gromov hyperbolic space, $\tilde{V}^{\prime}$ is provided with a path metric induced from the Riemannian metric of $\tilde{V}^{\prime}$. We take the metric completion of $\tilde{V}^{\prime}$ and get a geodesic space $\bar{V}^{\prime}$. The triangles in our space $\bar{V}^{\prime}$ are known to be thin only when they do not touch the boundary. Still, we can apply the same argument as the proof of the stability for $\bar{V}^{\prime}$, and can show that the geodesic connecting the endpoint of $\tilde{c}^{\prime}$ cannot touch the boundary since $\tilde{c}^{\prime}$ is at the distance more than $C^{-1} L$ from the boundary, and also that the stability holds.

Thus if $\tilde{c}^{\prime}$ is at the distance greater than $N$ from the ends of $\tilde{V}^{\prime}$, then $\tilde{c}^{\prime}$ can be homotoped to a geodesic arc fixing the endpoints within the $N$ neighbourhood of $\tilde{c}^{\prime}$, and projecting this to $V^{\prime}$, we obtain a closed geodesic arc (not necessarily a closed geodesic) homotopic to the pull-back of $c^{*}$ in $M$. Therefore, if we take $K_{0}$ and $L$ large enough so that the frontier of $V_{1} \cap V_{2}$ is at the distance greater than $C N$, we see that the pull back of $c^{*}$ can be homotoped to a closed geodesic arc hence cannot be null-homotopic.

Thus we have shown that no simple closed curve in $O$ is null-homotopic in $M$ if $K_{0}$ is large enough, and completed the proof of the first part of Theorem 3 .

It remains to show that two simple closed curves in $O$ which are not homotopic on $S$ are not homotopic in $M$. Suppose that two simple closed curves $c_{1}$ and $c_{2}$ in $S$ represent distinct points in $O$. As in the previous paragraph, we have quasi-geodesics $c_{1}^{\prime}, c_{2}^{\prime}$ homotopic to $c_{1}, c_{2}$ in $M$, which are obtained by pulling back the closed geodesics $c_{1}^{*}$ and $c_{2}^{*}$ in $V_{1} \cap V_{2}$ representing the free homotopy classes of $c_{1}$ and $c_{2}$. We consider their infinite lifts $\hat{c}_{1}^{\prime}, \hat{c}_{2}^{\prime}$ in $\tilde{V}^{\prime}$, which are $\left(C^{2}, 0\right)$-quasi-geodesic lines. By applying the same argument 
as above using the stability of quasi-geodesic lines instead of that of quasigeodesic arcs, we see that $\hat{c}_{1}^{\prime}, \hat{c}_{2}^{\prime}$ can be homotoped to geodesic lines $\hat{c}_{1}^{*}, \hat{c}_{2}^{*}$ in $\tilde{V}^{\prime}$ by a proper homotopy which doe not touch the boundary of $\tilde{V}^{\prime}$. By projecting them down to $M$, we get closed geodesics $c_{1}^{\prime *}, c_{2}^{\prime *}$, which must coincide since we assumed that $c_{1}$ and $c_{2}$ are homotopic in $M$. It follows that $c_{1}^{\prime}$ and $c_{2}^{\prime}$ are homotopic in $V^{\prime}$, which implies that $c_{1}^{*}$ and $c_{2}^{*}$ are homotopic in $V_{1} \cap V_{2} \cong S \times(0,1)$. This contradicts our assumption that $c_{1}$ and $c_{2}$ are not homotopic in $S$. This shows that $c_{1}$ and $c_{2}$ cannot be homotopic in $M$, and we have completed the proof of Theorem 3 .

Next we shall show that $\left\langle G_{1}, G_{2}\right\rangle$ has a non-empty domain of discontinuity on $\mathcal{P} \mathcal{M L}(S)$.

Theorem 4. Let $O$ be an open set in $\mathcal{P} \mathcal{M L}(S)$ as in Theorem 3. Then we have $\left\{g \in\left\langle G_{1}, G_{2}\right\rangle \mid g O \cap O \neq \emptyset\right\}=\{1\}$.

Proof. Suppose that $U:=g O \cap O$ is non-empty. Let $c$ be any simple closed curve in $U$. Then, there exists a simple closed curve $c^{\prime}$ in $O$ with $g\left(c^{\prime}\right)=c$. Since $\left\langle G_{1}, G_{2}\right\rangle$ acts on $\pi_{1}(M)$ trivially, we see that $c$ is freely homotopic to $c^{\prime}$ in $M$. Since $c$ and $c^{\prime}$ are contained in $O$, by Theorem 3, it follows that $c=c^{\prime}$. This shows that $g$ fixes every simple closed curve contained in $U$. Since the simple closed curves are dense in $U$, this implies that $g$ fixes $U$ pointwise. It is easy to see as follows that such an element in $\operatorname{MCG}(S)$ must be the identity. Since $g$ is not a torsion, it is either pseudo-Anosov or reducible. If $g$ is pseudo-Anosov, it fixes only two points in $\mathcal{P} \mathcal{M L}(S)$. If $g$ is reducible, some of its powers, $g^{p}$ is either partially pseudo-Anosov, i.e. , there is a subsurface $T$ of $S$ such that $g^{p} \mid T$ is pseudo-Anosov, or a product of nontrivial powers of Dehn twists along mutually disjoint essential simple closed curves $c_{1}, \cdots, c_{m}$ on $S$. In the first case, let $\lambda_{u}, \lambda_{s}$ be the unstable and stable laminations of $g^{p} \mid T$. Then $g^{p}$ fixes only a measured lamination whose supports contain either $\left|\lambda_{u}\right|$ or $\left|\lambda_{s}\right|$. Such laminations cannot constitute an open set. Therefore $g^{p}$ cannot fix all points in $U$. In the latter case, the fixed point set of the action of $g^{p}$ on the projective measured lamination space consists of those elements whose underlying geodesic laminations do not intersect $\cup c_{i}$ transversely. Such a subspace of $\mathcal{P} \mathcal{M L}(S)$ cannot contain a nonempty open set. Therefore $g^{p}$ cannot fix all points in $U$ in this case, too.

\section{ITERATION OF PSEUDO-ANOSOV MAP}

In the case when two handlebodies are pasted by an $n$-time iteration of a pseudo-Anosov map, we can have a region as in Theorems 3 and 4 , which gets larger and larger as $n \rightarrow \infty$ to cover the complement of the closure of meridians and isolated points as we shall see below.

Let $H_{1}$ and $H_{2}$ be handlebodies whose boundary is identified with a closed orientable surface $S$ of genus $>1$. Let $\phi: S \rightarrow S$ be a pseudo-Anosov map with a stable lamination $\mu_{\phi}$ and an unstable lamination $\lambda_{\phi}$. For each 
positive integer $n$, we consider the 3 -manifold $M_{n}=H_{1} \cup_{\phi^{n}} H_{2}$ obtained by identifying the boundaries of $H_{1}$ and $H_{2}$ via $\phi^{n}: \partial H_{1}=S \rightarrow S=\partial H_{2}$. As in the introduction, let $G_{1}$ be the subgroup of $\operatorname{Aut}(\mathcal{C C}(S))$ obtained as the image of the subgroup of $\operatorname{MCG}(S)$ consisting of those classes of autohomeomorphisms of $H_{1}$ which are homotopic to the identity in $H_{1}$. The other handlebody $H_{2}$ is regarded as embedded in $M_{n}$ as $H_{2}^{(n)}$ and $\partial H_{2}^{(n)}=$ $\partial H_{1}=S$. We let $G_{2}^{(n)}$ be the subgroup of the subgroup of $\operatorname{Aut}(\mathcal{C C}(S))$ obtained as the image of the subgroup of $\mathrm{MCG}(S)$ consisting of those classes of auto-homeomorphisms of $H_{2}^{(n)}$ which are homotopic to the identity in $H_{2}^{(n)}$. We denote by $G^{(n)}$ the subgroup of $\operatorname{Aut}(\mathcal{C C}(S))$ generated by $G_{1}$ and $G_{2}^{(n)}$.

In the measured lamination space $\mathcal{M L}\left(\partial H_{1}\right)$, we define the set of doubly incompressible laminations $\mathcal{D}\left(H_{1}\right)$ to be

$\mathcal{D}\left(H_{1}\right)=\left\{\lambda \in \mathcal{M L}\left(\partial H_{1}\right) \mid \exists \eta>0\right.$ such that $i(\lambda, m)>\eta$ for every meridian $\left.m\right\}$,

following Lecuire [14] and Kim-Lecuire-Ohshika [12]. We denote the projection of $\mathcal{D}\left(H_{1}\right)$ to $\mathcal{P} \mathcal{M L}\left(\partial H_{1}\right)$ by $\mathcal{P} \mathcal{D}\left(H_{1}\right)$. As was shown in Theorem 1.4 in Lecuire [15], the group $G_{1}$ acts on $\mathcal{D}\left(H_{1}\right)$ properly discontinuously. A smaller open set called the Masur domain is defined as follows. First we define $\mathcal{C}\left(H_{1}\right)$ by

$$
\begin{gathered}
\mathcal{C}\left(H_{1}\right)=\left\{c \in \mathcal{M L}\left(\partial H_{1}\right) \mid \text { the support of } c\right. \text { is a simple closed curve } \\
\text { bounding a disc in } \left.H_{1}\right\} .
\end{gathered}
$$

We then define the Masur domain to be

$$
\mathcal{M}\left(H_{1}\right)=\left\{\lambda \in \mathcal{M L}(S) \mid i(\lambda, \mu)>0 \text { for any } \mu \in \overline{C\left(H_{1}\right)}\right\} .
$$

We note that $\mathcal{D}\left(H_{1}\right)$ contains $\mathcal{M}\left(H_{1}\right)$ and that any arational lamination in $\mathcal{D}\left(H_{1}\right)$ is also contained in $\mathcal{M}\left(H_{1}\right)$ (see [14, Lemmas 3.1 and 3.4]). Since $\mathcal{D}\left(H_{1}\right)$ contains the projectivised Masur domain of $H_{1}$ which was proved to have full measure in $\mathcal{P} \mathcal{M L}(S)$ by Kerckhoff [11, $\mathcal{P} \mathcal{D}\left(H_{1}\right)$ also has full measure.

Theorem 5. Assume that $\lambda_{\phi}$ is contained in $\mathcal{M}\left(H_{1}\right)$ and $\mu_{\phi}$ is contained in $\mathcal{M}\left(H_{2}\right)$. Then for any projective lamination $[\lambda]$ in $\mathcal{P} \mathcal{D}\left(H_{1}\right) \backslash G_{1}\left[\lambda_{\phi}\right]$, there are an open neighbourhood $U$ and $n_{0} \in \mathbb{N}$ such that for any $n \geq n_{0}$, no simple closed curve whose projective class is contained in $U$ is null-homotopic, and $\left\{g \in G^{(n)} \mid g U \cap U \neq \emptyset\right\}$ is finite.

Proof. Let $\iota_{n}: H_{1} \rightarrow M_{n}$ be the inclusion. Let $\phi_{n}: \pi_{1}\left(M_{n}\right) \rightarrow \mathrm{PSL}_{2} \mathbb{C}$ be a representation corresponding to the hyperbolic structure on $M_{n}$. In $\S 5$ of Namazi-Souto [28] it was proved that under our assumptions, $\left\{\phi_{n} \circ\right.$ $\left.\iota_{n}\right\}$ converges up to conjugations, where we continue to denote by $\iota_{n}$ the homomorphism $\pi_{1}\left(H_{1}\right) \rightarrow \pi_{1}\left(M_{n}\right)$ induced by $\iota_{n}$. Note that we see by [14. Lemma 3.4] that the homeomorphism $\phi$ is generic in the sense of 28, Definition 2.1] i.e., the unstable lamination $\lambda_{\phi}$ is not a limit, in $\mathcal{P} \mathcal{M L}\left(\partial H_{1}\right)$, 
of meridians of $H_{1}$, and the stable lamination $\mu_{\phi}$ is not a limit of meridians of $\mathrm{H}_{2}$.

Fix some nontrivial element $\gamma \in \pi_{1}\left(H_{1}\right)$. For any sufficiently large $n$, the element $\iota_{n}(\gamma)$ represents a non-trivial element of $\pi_{1}\left(M_{n}\right)$ (see 28, Lemma 6.1]). Let $\gamma_{n}^{*}$ be a closed geodesic in $M_{n}$ representing $\gamma$ in $\pi_{1}\left(M_{n}\right)$. Since $\phi_{n} \circ \iota_{n}$ converges up to conjugations, the length of $\gamma_{n}^{*}$ is bounded as $n \rightarrow \infty$ and $\gamma_{n}^{*}$ converges geometrically to the projection in the geometric limit of some closed geodesic in the algebraic limit. Hence the distance between $\gamma_{n}^{*}$ and a spine of $H_{1}$ is bounded. Therefore, if we take a base point $x_{n}$ on $\gamma_{n}^{*}$, then the geometric limit of $\left(M_{n}, x_{n}\right)$ is a hyperbolic 3-manifold whose fundamental group is identified with $\pi_{1}\left(H_{1}\right)$. We denote this geometric limit by $M_{\infty}$. Namazi-Souto showed that this $M_{\infty}$ coincides with the hyperbolic 3-manifold corresponding to the limit $\phi_{\infty}$ of $\left\{\phi_{n} \circ \iota_{n}\right\}$ (see [28, Theorem 5.2]). This hyperbolic 3-manifold has a unique end, which has ending lamination corresponding to the limit of meridians of $\mathrm{H}_{2}$ regarded as a simple closed curve on $\partial H_{1}$ by pasting map $\phi^{-n}$ as $n \rightarrow \infty$, which coincides with $\lambda_{\phi}$. (The limit does not depend on the choice of meridian.) By the uniqueness of ending lamination and Theorem 5.1 in Lecuire [14, every lamination contained in $\mathcal{P} \mathcal{D}\left(H_{1}\right) \backslash G_{1}\left[\lambda_{\phi}\right]$ is realisable by a pleated surface homotopic to the inclusion of $\partial H_{1}$.

Now, let $[\lambda]$ be a projective lamination contained in $\mathcal{P} \mathcal{D}\left(H_{1}\right) \backslash G_{1}\left[\lambda_{\phi}\right]$ Since $G_{1}$ acts on $\mathcal{P} \mathcal{D}\left(H_{1}\right)$ properly discontinuously, $\mathcal{P} \mathcal{D}\left(H_{1}\right) \backslash G_{1}\left[\lambda_{\phi}\right]$ is an open set, and hence there is a neighbourhood $U$ of $[\lambda]$ which is contained in $\mathcal{P} \mathcal{D}\left(H_{1}\right) \backslash G_{1}\left[\lambda_{\phi}\right]$. We take $U$ so that its closure $\bar{U}$ is compact and is also contained in $\mathcal{P} \mathcal{D}\left(H_{1}\right) \backslash G_{1}\left[\lambda_{\phi}\right]$. Then every simple closed curve in $\bar{U}$ is realised by a pleated surface in $M_{\infty}$. We shall show that there is $n_{0} \in \mathbb{N}$ such that for every $n \geq n_{0}$ and every simple closed curve in $U$ represents a non-trivial class in $\pi_{1}\left(M_{n}\right)$.

Suppose not. Then there is a sequence of simple closed curves with $\left[\gamma_{n}\right]$ contained in $U$ such that $\gamma_{n}$ is null-homotopic in $M_{n}$. Taking a subsequence, $\left[\gamma_{n}\right]$ converges to a projective lamination $[\mu]$ passing to a subsequence. The projective lamination $[\mu]$ is contained in $\bar{U}$; hence by our choice of $U$, it is contained in $\mathcal{P} \mathcal{D}\left(H_{1}\right) \backslash G_{1}\left[\lambda_{\phi}\right]$. Now, by the same argument as $\S 6$ in Ohshika [30, this implies that there exists a neighbourhood $V$ of $[\mu]$ and $N \in \mathbb{N}$ such that every lamination in $V$ is realisable in $M_{n}$ for $n \geq N$. Indeed, since $\mu$ is realisable in the geometric limit $M_{\infty}$, there is a train track $\tau$ with small curvature carrying $\mu$ such that every measured lamination carried by $\tau$ is realised in a fixed neighbourhood of the realisation of $\mu$. By pulling back this to $M_{n}$ by an approximate isometry, we see that we have a neighbourhood as above.

Now, the existence of such $V$ contradicts the assumption that $\left[\gamma_{n}\right]$ is null-homotopic in $M_{n}$. Thus we have shown that there is a neighbourhood $U$ of $\lambda$ such that for every $n \geq n_{0}$ and every simple closed curve in $U$ represents a non-trivial class in $\pi_{1}\left(M_{n}\right)$. Repeating the argument in the proof of Theorem [3, we can also show that by letting $U$ smaller and $n_{0}$ 
larger if necessary, no two distinct simple closed curves in $U$ are homotopic in $M_{n}$ for $n \geq n_{0}$.

Moreover, by the same argument as in the proof of Theorem 4, we can show that by letting $U$ be smaller and $n_{0}$ larger, we have the condition $\left\{g \in G^{(n)} \mid g U \cap U \neq \emptyset\right\}$ is finite for every $n \geq n_{0}$.

\section{REFERENCES}

[1] R. Benedetti and C. Petronio, Lectures on hyperbolic geometry, Universitext. Springer-Verlag, Berlin, 1992. xiv+330 pp.

[2] B. Bowditch, Tight geodesics in the curve complex, Invent. Math. 171(2008), 281-300.

[3] J. Brock, Y. Minsky, H. Namazi and J. Souto, Bounded combinatorics and uniform models for hyperbolic 3-manifolds, draft.

[4] M. Bridson and A. Haefliger, Metric spaces of non-positive curvature, Grundlehren der Mathematischen Wissenschaften 319, Springer-Verlag, Berlin, 1999. xxii+643 pp.

[5] R.D. Canary,D.B.A. Epstein, and P. Green, Notes on notes of Thurston, Analytical and geometric aspects of hyperbolic space (Coventry/Durham, 1984), pp.3-92, London Math. Soc. Lecture Note Ser., 111, Cambridge Univ. Press, Cambridge, 1987.

[6] M. Coornaert, T. Delzant, and A. Papadopoulos, Géométrie et théorie des groupes. Les groupes hyperboliques de Gromov, Lecture Notes in Mathematics, 1441 SpringerVerlag, Berlin, 1990. $\mathrm{x}+165$ pp.

[7] C. Gordon, Problems, Workshop on Heegaard Splittings, 401-411, Geom. Topol. Monogr. 12, Geom. Topol. Publ., Coventry, 2007.

[8] M. Gromov, Metric structures for Riemannian and non-Riemannian spaces. With appendices by M. Katz, P. Pansu and S. Semmes. Translated from the French by Sean Michael Bates. Progress in Mathematics, 152. Birkhäuser Boston, Inc., Boston, MA, 1999. $\mathrm{xx}+585 \mathrm{pp}$.

[9] N.V. Ivanov, Mapping class groups, Handbook of geometric topology, 523-633, NorthHolland, Amsterdam, 2002.

[10] J. Johnson, Mapping class groups of medium distance Heegaard splittings, Proc. Amer. Math. Soc. 138 (2010), 4529-4535.

[11] S. Kerckhoff, The measure of the limit set of the handlebody group, Topology 29 (1990), 27-40.

[12] I. Kim, C. Lecuire and K. Ohshika, Convergence of freely decomposable Kleinian groups, preprint.

[13] M. Korkmaz, Automorphisms of complexes of curves on punctured spheres and on punctured tori, Topology Appl., 95 (2) (1999) 85-111.

[14] C. Lecuire, An extension of the Masur domain, Spaces of Kleinian groups, 49-73, London Math. Soc. Lecture Note Ser. 329, Cambridge Univ. Press, Cambridge, 2006.

[15] C. Lecuire, Bending map and strong convergence, preprint, available at http://www.math.univ-toulouse.fr/ lecuire/properness.pdf

[16] D. Lee and M. Sakuma, Simple loops on 2-bridge spheres in 2-bridge link complements, Electron. Res. Announc. Math. Sci. 18 (2011), 97-111.

[17] D. Lee and M. Sakuma, Epimorphisms between 2-bridge link groups: Homotopically trivial simple loops on 2-bridge spheres, Proc. London Math. Soc. 104 (2012), 359386.

[18] D. Lee and M. Sakuma, Homotopically equivalent simple loops on 2-bridge spheres in 2-bridge link complements (I), (II) and (III), to appear in Geometriae Dedicata.

[19] D. Lee and M. Sakuma, A variation of McShane's identity for 2-bridge links, Geometry \& Topology 17 (2013), 2061-2101.

[20] F. Luo, Automorphisms of the complex of curves, Topology, 39 (2) (2000) 283-298. 
[21] H. Masur and Y. Minsky, Geometry of the curve complex I: Hyperbolicity, Invent. Math. 138 (1999), 103-149.

[22] H. Masur and Y. Minsky, Geometry of the complex of curves. II. Hierarchical structure, Geom. Funct. Anal. 10 (2000), 902-974.

[23] H. Masur and Y. Minsky, Quasiconvexity in the curve complex, In the tradition of Ahlfors and Bers, III, 309-320, Contemp. Math. 355, Amer. Math. Soc., Providence, RI, 2004.

[24] Y. Minsky, On rigidity, limit sets, and end invariants of hyperbolic 3-manifolds, J. Amer. Math. Soc. 7 (1994), 539-588.

[25] H. Namazi, Heeggard splittings and hyperbolic geometry, Thesis, Stony Brook University, 2005

[26] H. Namazi, Big Heegaard distance implies finite mapping class group, Topology and its Appl. 154 (2007), 2939-2949

[27] H. Namazi, Heegaard splittings with bounded combinatorics, in preparation

[28] H. Namazi and J. Souto, Heegaard splittings and pseudo-Anosov maps, Geom. Funct. Anal. 19 (2009), no. 4, 1195-1228.

[29] H. Namazi and J. Souto, Non-realizability and ending laminations: proof of the density conjecture. Acta Math. 209 (2012), no. 2, 323-395.

[30] K. Ohshika, Kleinian groups which are limits of geometrically finite groups, Mem. Amer. Math. Soc. 177 (2005), no. 834

[31] K. Ohshika, Discrete groups, Translated from the 1998 Japanese original by the author. Translations of Mathematical Monographs 207, Iwanami Series in Modern Mathematics. American Mathematical Society, Providence, RI, 2002. x+193 pp.

[32] K. Ohshika, Realising end invariants by limits of minimally parabolic, geometrically finite groups, Geom. and Topol. 15 (2011) 827-890.

[33] T. Ohtsuki, R. Riley, and M. Sakuma, Epimorphisms between 2-bridge link groups, Geom. Topol. Monogr. 14 (2008), 417-450.

[34] J-P. Otal, Courants géodésiques et produits libres, Thèse d'Etat, Université de ParisSud, Orsay (1988).

[35] M. Sakuma, Problem session, In "Geometric and analytic approaches to representations of a group and representation spaces", R.I.M.S. Kokyuroku 1777 (2012), $110-112$.

[36] W. Thurston, The geometry and topology of three-manifolds, lecture notes, available at http://library.msri.org/books/gt3m/

[37] G. Tian, A pinching theorem on manifolds with negative curvature, preprint.

Department of Mathematics, Graduate School of Science, Osaka UniverSITY, TOYONAKA, OSAKA, 560-0043, JAPAN

E-mail address: ohshika@math.sci.osaka-u.ac.jp

Department of Mathematics, Graduate School of Science, Hiroshima UnivERSity, Higashi-Hiroshima, 739-8526, JAPAN

E-mail address: sakuma@math.sci.hiroshima-u.ac.jp 